\title{
Anode bottom burnout shape and velocity field investigation in a high amperage electrolysis cell
}

\author{
V. Bojarevics ${ }^{1}$, E. Radionov ${ }^{2}$ and Y. Tretiyakov ${ }^{2}$ \\ ${ }^{1}$ University of Greenwich, UK \\ ${ }^{2}$ Rusal ITC, Russia
}

Keywords: magnetohydrodynamics of liquid metals, model validation, aluminium electrolysis cells, RUSAL technology

Long term burnout shape of the anode bottom is believed to reflect the quasi-stationary liquid metal interface dome-shaped deformation. The shape profiles and their interplay affect the MHD stability, the velocity fields, set up of new anodes and electrical efficiency of the cell. Spent prebake anode bottom profiles were systematically measured at Rusal $309 \mathrm{kA}$ cell to obtain the overall view of the liquid metal deformation. The magnetic field distribution and velocities of liquid metal flow were measured in order to obtain a more complete characterization of the cell. The results are compared to the modelling results using the specialised MHDVALDIS software giving the insight to the cell dynamics.

\section{Introduction}

Industrial aluminium production cells are highly optimised for magnetic field and electric current distribution in order to avoid MHD instabilities, increase the energy savings and to maintain a stable electrolytic process during normal operation. RUSAL company has spent considerable effort in the direction to reduce the power consumption and increase the productivity over a number of years [1]. Mathematical modelling has become a primary design and optimization tool of aluminium electrolysis cells. There are several commercial packages available to be used as modelling tools to model and possibly improve the performance of the commercial cells. The advantage of specialised commercial software packages is that they are being continuously developed and maintained to meet the interests of the aluminium industry. For instance, the universal package ANSYS is successfully used for the thermo-electric and mechanical modelling of the reduction cells [2,3]. There are attempts to use the ANSYS/CFX package for the hydrodynamic and magnetic simulations [4-6]. The velocity field has been successfully modelled and validated against experiments using the ESTER/PHOENICS [3,7]. Similar validation of the velocity fields, magnetic fields, electric current distribution and the wave oscillation frequencies were achieved using the specialised package MHD-VALDIS [3,8,9] using more streamlined approach to save time and effort for the commercial implementation. This software is based on the full MHD model of the electrolysis cell applied to a particular commercial cell replicated in the model using the specialised input suitable for fast implementation. Briefly, the full model computes time-dependent currents, voltages, magnetic fields, bath-metal interface shape and turbulent magnetically driven flow in the bath and liquid metal [10,11]. As an example, the Figure 1 shows the RUSAL OA-300M2 cell pot-line and the zoomed view to all the conductors that are part of the simulation. The conductors are coloured by their computed currents, current densities or by the bar temperatures, as required. The temperatures of the bus bars are adjusted according to the computed Joule heating, heat transfer to the neighbour bars and losses to the air. The electric current in the individual anodes is computed at all times following the wave development on the interface between liquid layers. The dome shaped time average deformation of the metal surface is projected to the bottom of the whole anode block to account for the anode bottom burn-out effect for a constant ACD, except in the case of disturbance, such as an anode change operation. The electric current distribution in the liquid zone is computed from all the bus bar network connected to the anodes and cathode collectors, coupled with the cell interior details like the ledge profile, bottom shape, collector connections, electrolyte channels and the electrochemical voltage drop. The magnetic field is optionally recomputed at all times in order to follow the full magnetohydrodynamic wave and flow development. The detailed representation of the cell steel elements adds non-linearity to the overall magnetic field properties. The MHD response of different commercial cells is quite variable depending on their design (side- to-side, end-to-end, Soderberg, etc.), the line amperage, operational practices (tapping, anode change, bottom erosion, alumina feeding), and many other factors. The present 
investigation focuses on a particular cell type at the RUSAL plant where the measurement has been recently obtained for the $309 \mathrm{kA}$ cell. The setup of the model is facilitated by the specialised inputs of the MHD software, however the interpretation of the results is not an exact science. The use of input material parameter values, their temperature dependence, the cell aging process and the complexity of the operational practice makes the precise inputs difficult and requires creative mind to find the right solutions suit practical demand of industry. For instance, the electric current distribution is considerably affected by the influence of the initial shape and position of an anode and the curvature of the aluminium [10], effects of the gas bubble concentration at the anode bottom [13], and the various contact resistances in the cathode assembly [14] and anode connectors. These features are available as the initial setup parameters within the software. The dynamic nature of the software tool permits to simulate the so-called MHD stability limit of the cell either following the total cell voltage oscillation or the anode current fluctuations. The effects of all anode rise or lowering can be simulated to predict the dynamic effects [15]. The present paper considers the RUSAL $309 \mathrm{kA}$ cell in detail and attempts to reflect the modelling efforts required to gain understanding of the measurement and modelling similarities and discrepancies.

\section{The comparison of the measurements and numerical results}

The full arrangement of the test cell in the potline is shown in the Figure 1 exactly as implemented in the MHDVALDIS software solution. The model includes the steel construction elements (shown here in grey) which are discretised in finite elements to compute the non-linear magnetization within the steel. In this case the colour of the bars corresponds to the current value in it. The electric current redistributes dynamically, meaning that the currents in the anodes are following the electrolyte/metal interface variation. Knowing the current distribution in the all surrounding bus bars, the neighbour cells, in the liquid metal and bath, and the magnetization of the steel, it is possible to reproduce the full magnetic field in any space position, including the liquid metal. The measurements of the magnetic field at the RUSAL plant were conducted using the 3-component Hall probe MAL 3.2 specifically designed for the use in the hostile environment, see the picture in the Figure 2. Some comparison results are shown in the Figure to reflect that the modelling is coming close, but not exactly replicating the measurements. In order to address the discrepancies the model was adjusted, first, attempting to increase the accuracy of the steel element discretisation, see the Figure 3. The finer mesh of the steel representation gives the improvement in the appearance and smoothness of the magnetic field distribution within the ferromagnetic parts. The convergence of the non-linear magnetization distribution requires about 2 hours for the course mesh (100k elements), 6 hours for the refined $(250 \mathrm{k})$ mesh and about 2 weeks for an extreme (1000k) mesh. However the magnetic field distribution in the liquid metal, where it is of the prime importance, remains practically the same, Figure 2, which leads to the conclusion that the moderately refined mesh of $250 \mathrm{k}$ elements is suitable for modelling. The second attempt to improve the field used the test of a different non-linear magnetization curve, as shown in the Figure 4. The curve M1(H) (left in the figure) is commonly used to achieve a better convergence rate by eliminating the sharp drop of $\mathrm{M}$ in the region of small field magnitudes. The second curve $\mathrm{M} 2(\mathrm{H})$ is a typical curve used for mild steels. The resulting magnetic permeability as computed for the fully converged cases are shown in the Figure 5. And again, the magnetic field distribution within the liquid metal is very similar for the both cases (Figure 2). The final magnetic field used for the velocity calculation is shown in the Figure 6 to compare the cases with the steel elements and without them. It is evident that neglecting the magnetized steel leads to excessive magnetic field values, particularly for the vertical $\mathrm{Bz}$ component, which is essential for the MHD stability and velocity calculations. The top superstructure shown in the Figure 3 contributes to the Bz component magnetic field distribution in the liquid metal.

The velocity field in the liquid metal was measured using the iron rod dissolution technique, see for instance [7,16]. The calibration curves were obtained at RUSAL using the rotating vessel of a given speed and time interval of immersion. An example of the rods used is shown in the Figure 7. The measurements are performed at two representative cells in a stable operation. The velocity measurements are represented in the Figure 7 , reflecting the directions and the magnitudes in the measurement locations. The respective numerical results are shown in the Figure 8 for two different values of the average ledge thickness at the bottom of the liquid volume. 
The ledge at the carbon bottom restricts the electric current passage and changes the distribution of the horizontal currents in the liquid metal. The Figure 9 illustrates the difference in the current distribution using a moderate $15 \mathrm{~cm}$ ledge all around the cell perimeter, and the extremely thin ledge of $1 \mathrm{~cm}$ only. It is worth mentioning that in experimenting with the ledge thickness effects, the result for a thick ledge of $30 \mathrm{~cm}$ (going below the anode shadow) was critical to lead to an unstable MHD waving. As seen from the Figure 8 and Figure 7 comparison, the ledge in the actual cell most probably is somewhere in between the cases of 15 and $1 \mathrm{~cm}$. The measured velocity field is reasonably close to the $15 \mathrm{~cm}$ ledge case both in directions and magnitudes of the velocities. The minor discrepancies could be attributed to the variation of the ledge in local positions. The software includes a possibility to prescribe the variable ledge profile if available from the measurements to obtain a more precise match in the velocities and the wave stability.

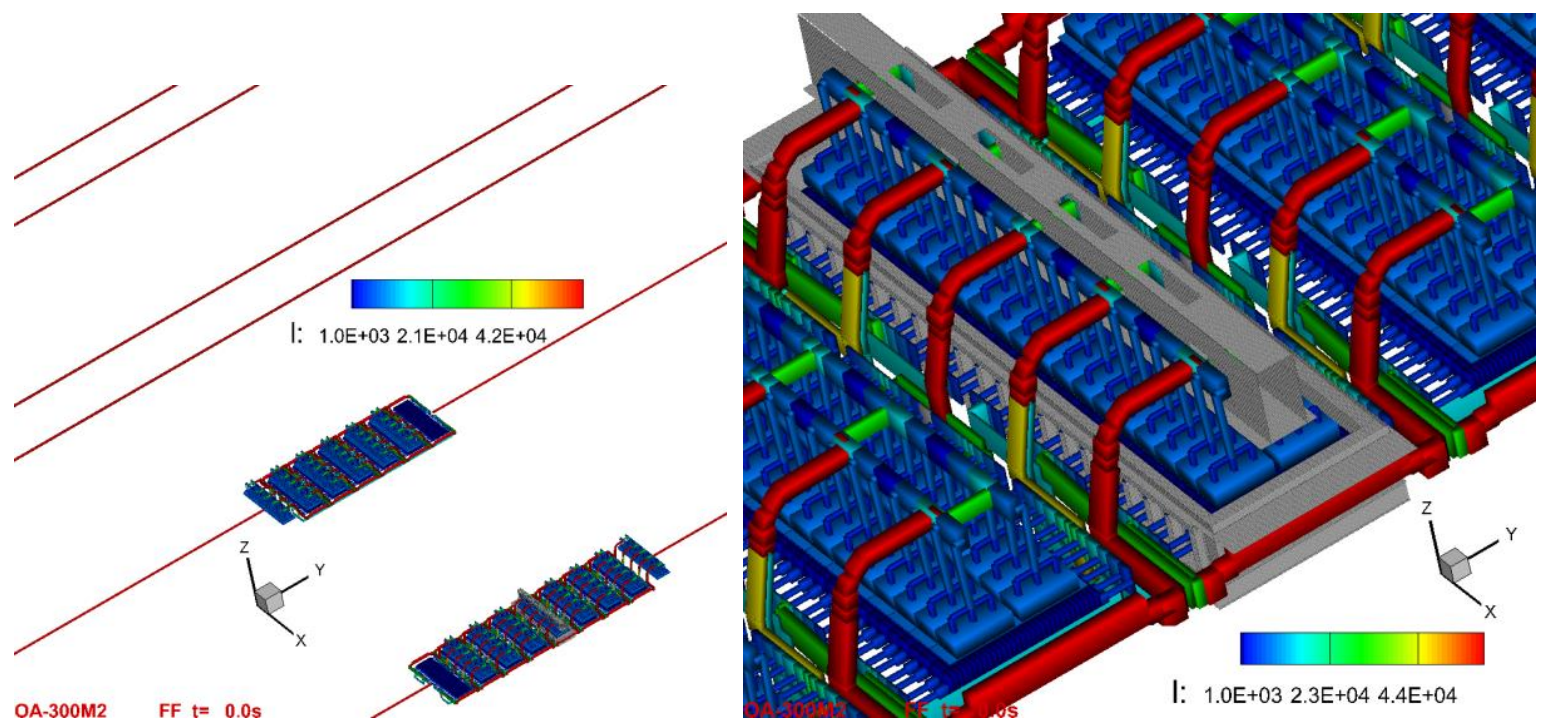

Figure 1. Full view with the neighbour lines and the zoom-in model of the $309 \mathrm{kA}$ cell.
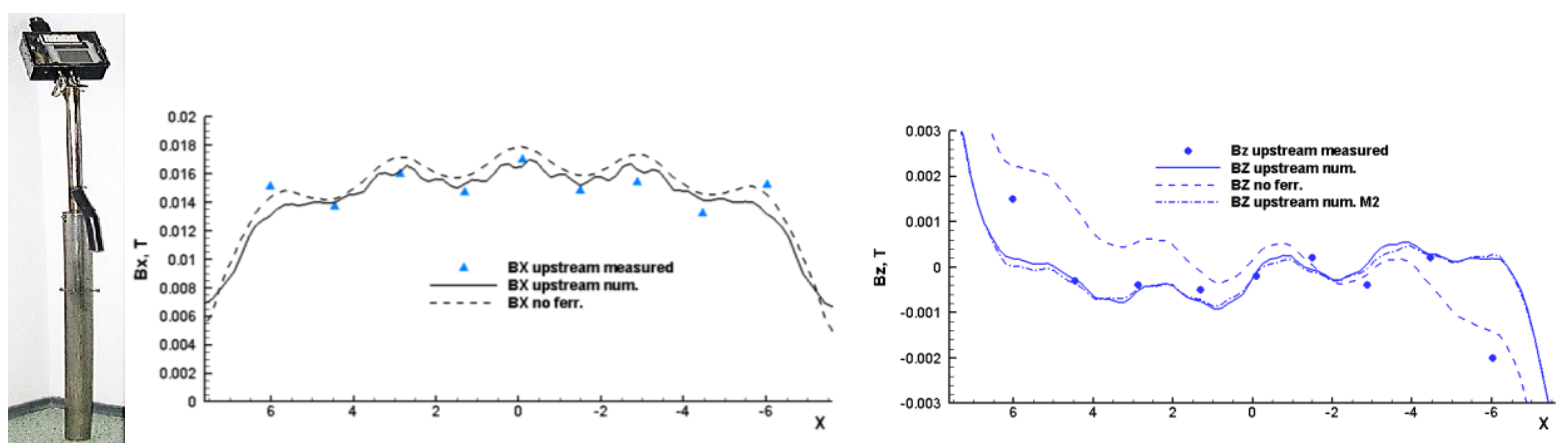

Figure 2. Magnetic field probe MAL-3.2 and the measurements compared to the numerical model of the $309 \mathrm{kA}$ cell. 

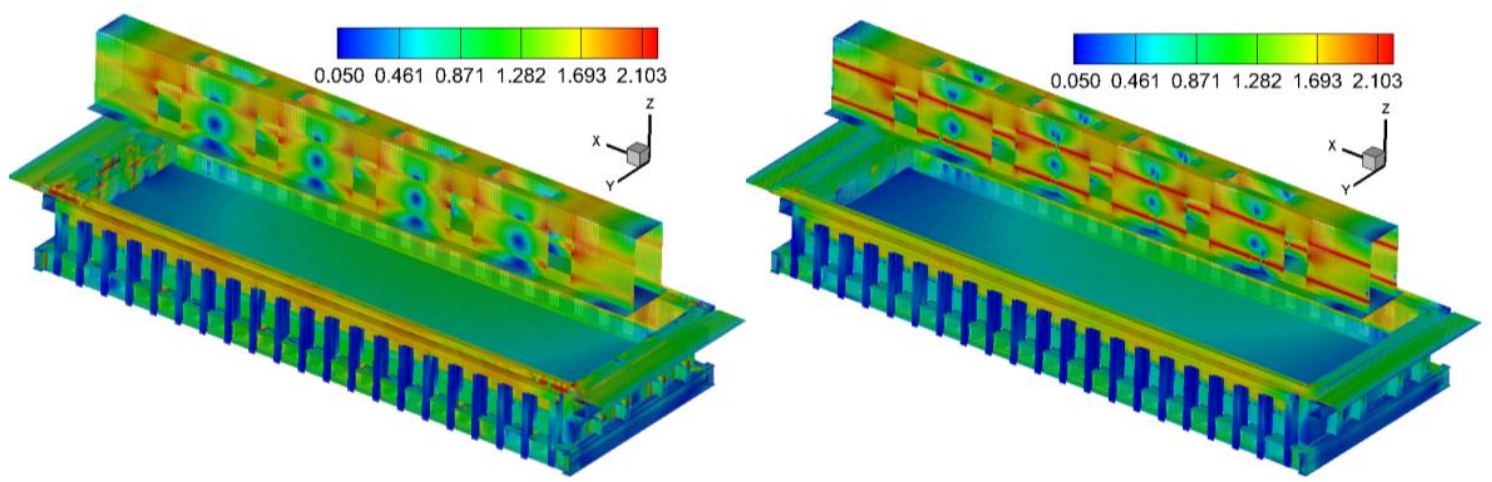

Figure 3. Magnetic field within the steel elements computed for the course mesh (100k elements) and fine mesh (250k elements).
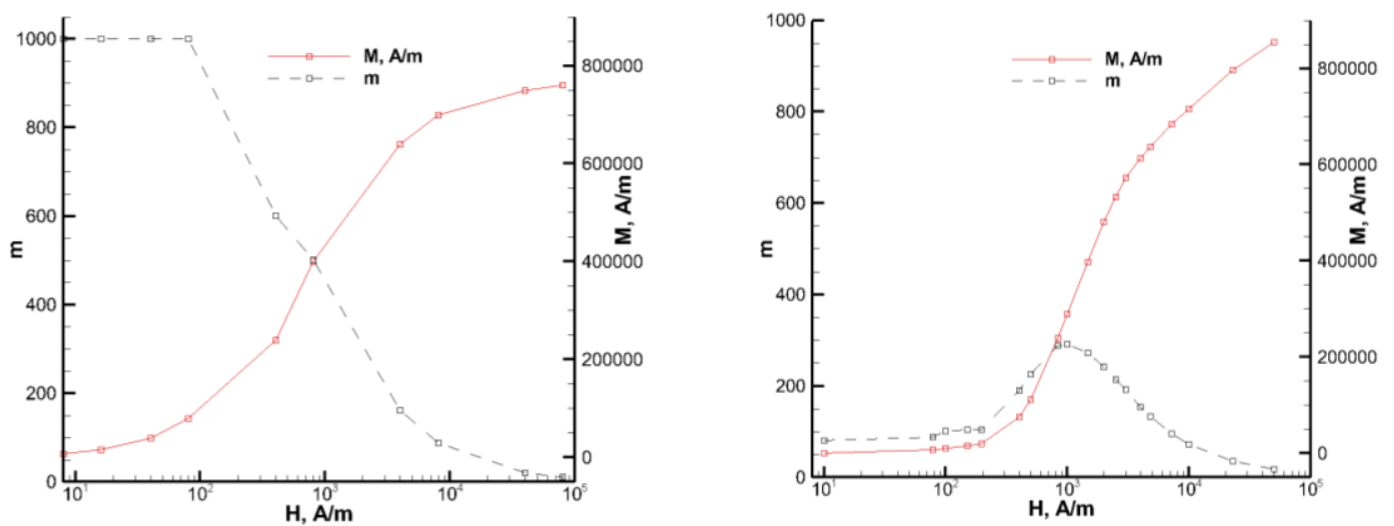

Figure 4. Magnetic permeability $\mathrm{m}(\mathrm{H})$ and magnetization $\mathrm{M}(\mathrm{H})$ curves used to model the $309 \mathrm{kA}$ cell: $\mathrm{M} 1$ (left) and M2 (right).
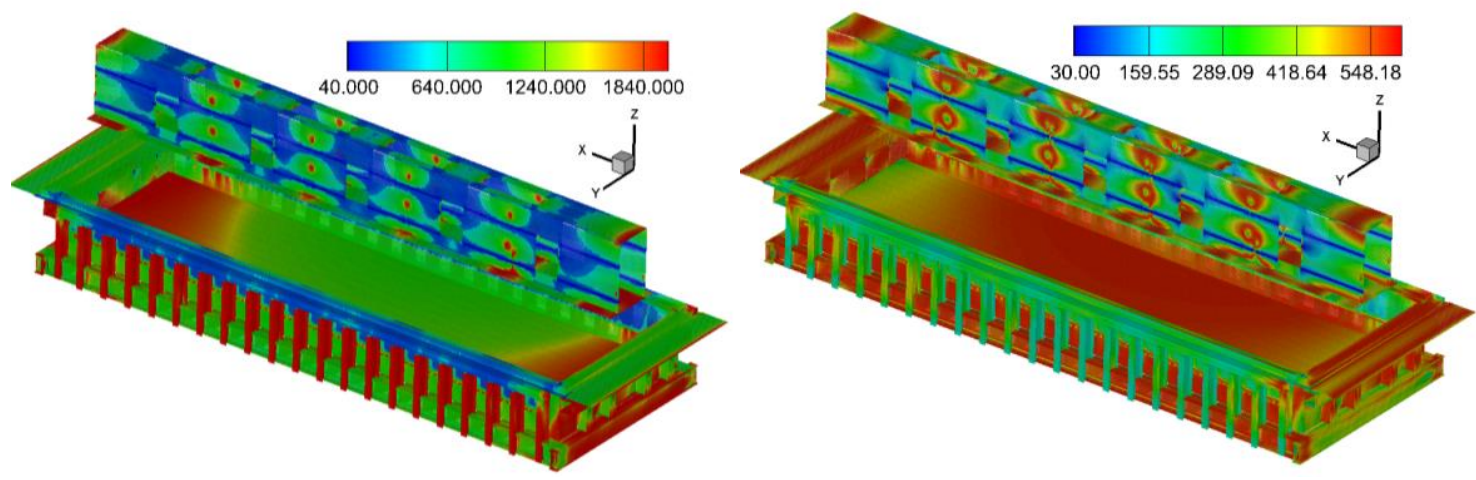

Figure 5. Magnetic permeability in the steel elements computed with the two magnetization curves: M1 (left) and M2 (right). 

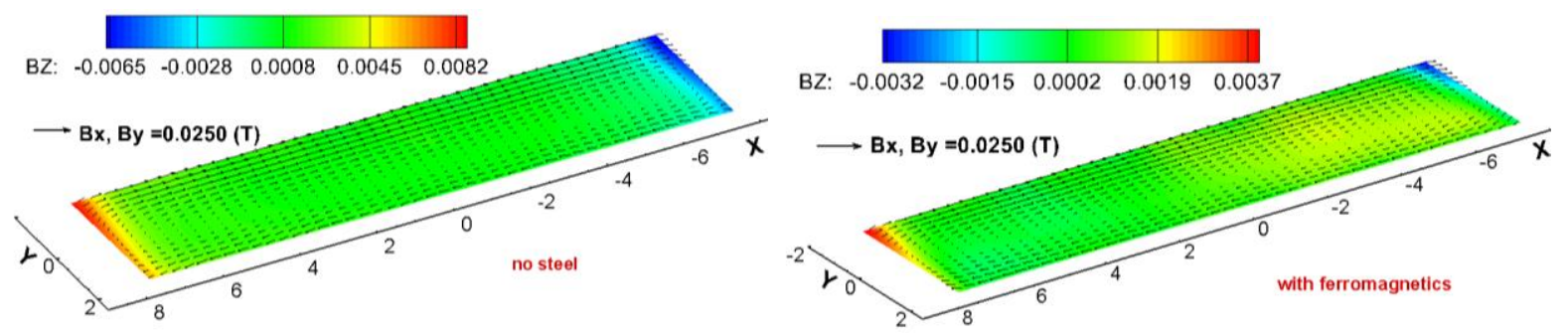

Figure 6. Comparison of the magnetic field in the liquid metal computed with and without the steel elements.

The third measurement series were performed to obtain the all anode bottom profile overall distribution for the full cell. It is well recognised that the 'dome' shape of the magnetically compressed liquid metal leads to the gradual selective burnout of the anode bottom, so that the local ACD remains constant [3,4,12]. The series of measurements at Rusal for 7 cells used the freshly extracted anodes from a stable operating cells. Each anode was positioned on a horizontal surface and the deviation of the bottom was measured near the central position and at the external edge, however some distance away from the bubble erosion curvature. The measurements were repeated for all 40 anodes to give the distribution of the anode bottom surface shown in the Figure 10 (left). The right side of the Figure 10 shows the numerically computed dome shape reflected on the anode bottom, which ensures the constant ACD operation conditions for the cell to be modelled. The comparison shows a reasonably good correspondence between the predicted anode bottom and the actually measured one. There is a clear asymmetry between the upstream and downstream cell sides, and the magnitude of the dome height is very close. The minor differences can be explained by the fact that actual cell has relatively new set anodes in the normal operational practice, which takes about 4-7 days for the anode to assume the final shape [10].
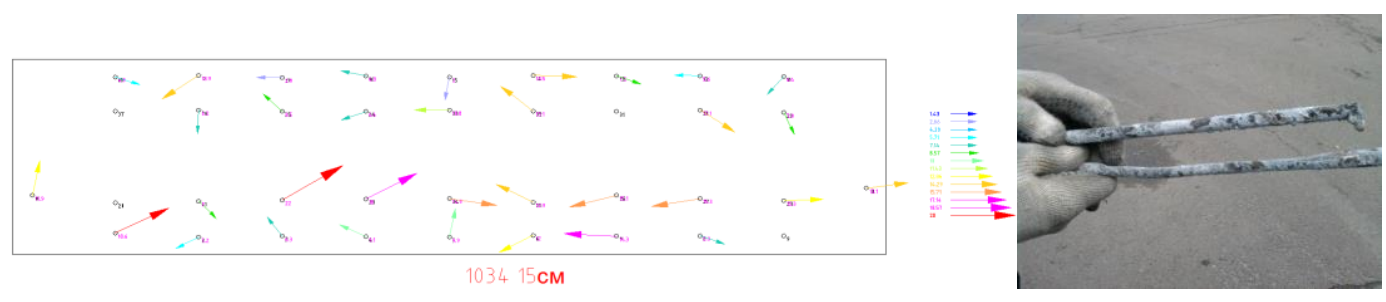

Figure 7. The measured velocity field in liquid metal for the $309 \mathrm{kA}$ cell and the example of used iron measurement rods.
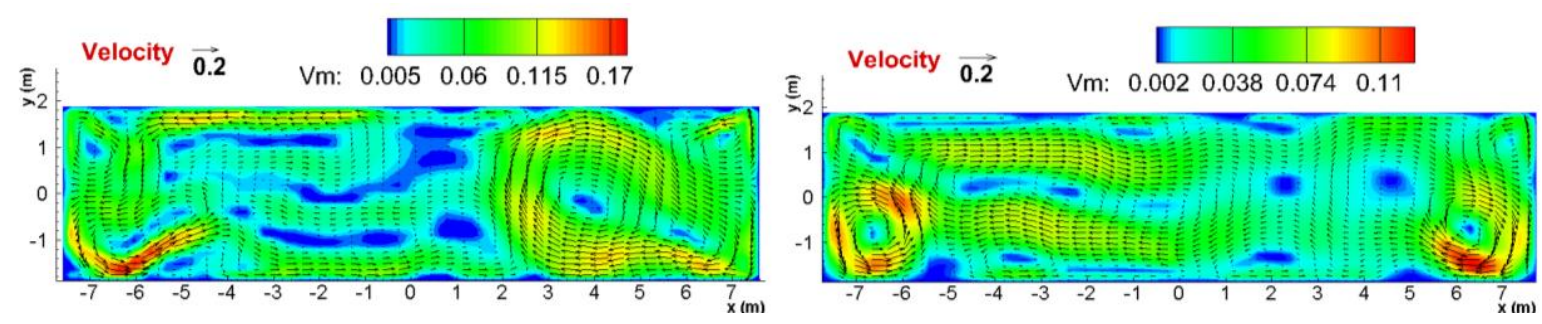

Figure 8 . The numerically computed velocity field in liquid metal for the average ledge thickness $0.15 \mathrm{~m}$ (left) and $0.01 \mathrm{~m}$ (right).
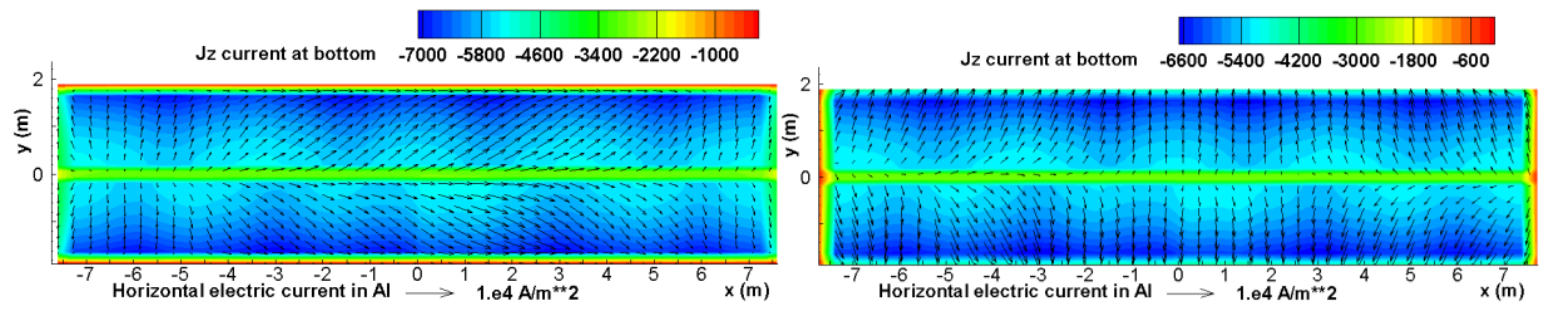
Figure 9. The numerically computed electric current in liquid metal for the ledge thickness $0.15 \mathrm{~m}$ and $0.01 \mathrm{~m}$.
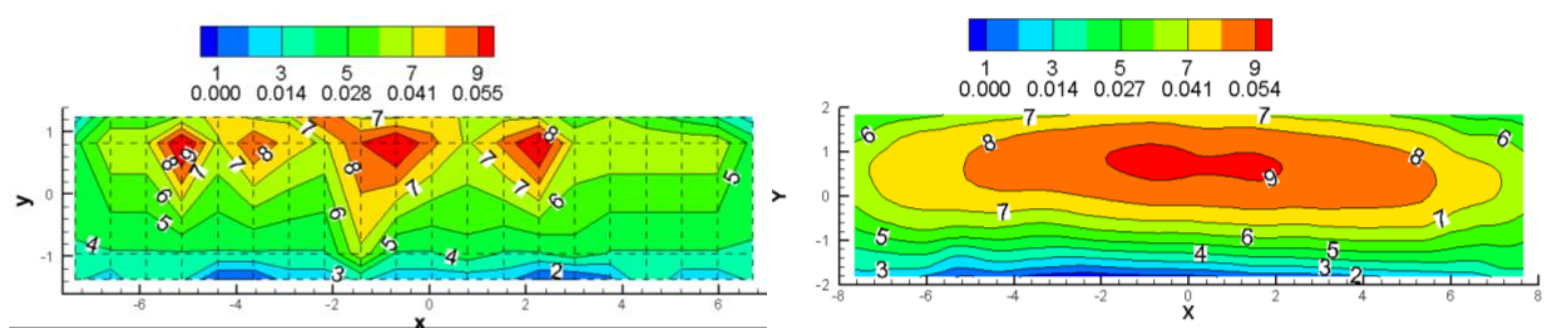

Figure 10. Anode bottom burnout: measured and numerical for the 309 kA cell.

The last measurement tests were dedicated to determine the MHD stability limit for the cell by using the all anode lowering technique and recording the voltage oscillation onset and their frequency. The normal cell operates at about $3.95 \mathrm{~V}$ potential drop. During the tests gradually lowering the anodes it was observed that harmonic oscillations appeared in the voltage reading when the total voltage drop approached about $3.5 \mathrm{~V}$. The observed frequencies were in the range $0.02-0.07 \mathrm{~Hz}$. The corresponding numerical simulations using the full dynamic solution lead to the results shown in the Figure 11. The simulation is started by establishing first a stationary flow, the anode bottom burnout and the respective current distribution. Then a perturbation of the longitudinal gravity wave of a small sloshing amplitude $(5 \mathrm{~mm})$ is applied to induce waving. For a stable operating cell at the ACD $=0.05 \mathrm{~m}$, this leads to a fast damping of the perturbation. When the ACD was reduced to $0.04 \mathrm{~m}, 0.038 \mathrm{~m}$ and $0.036 \mathrm{~m}$, it was found that the cell responds by increasing the wave perturbation amplitude with the decrease of the ACD. At the ACD $=0.036 \mathrm{~m}$, the wave gradually grew in amplitude to reach the contact to the anode bottom and short circuit locally. The lowest ACD when the stable, but slow damping was still observed was $0.037 \mathrm{~m}$, corresponding approximately to $3.7 \mathrm{~V}$ total voltage drop (Figure 11). The computed wave contained two distinct frequencies of about 0.025 and $0.047 \mathrm{~Hz}$, which are within the range observed in the experiment by lowering the anodes.
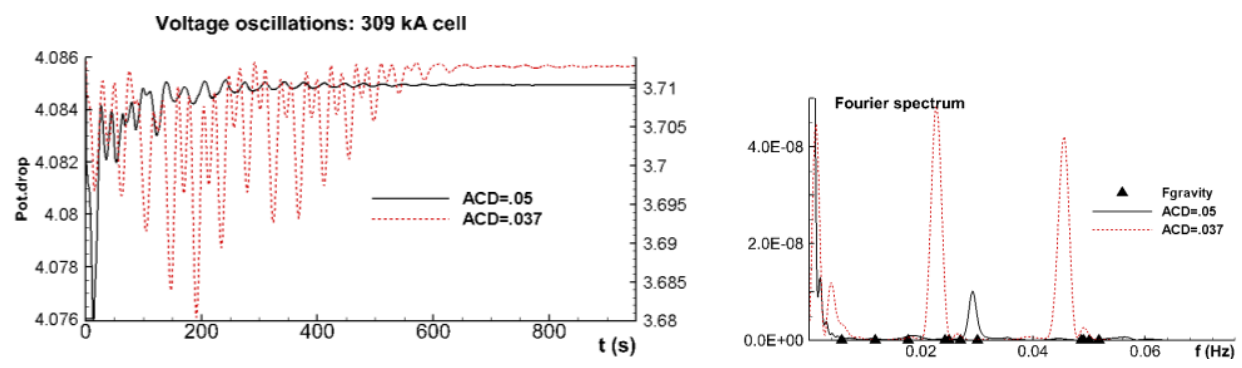

Figure 11. The numerically computed voltage oscillations following the initial perturbation of the interface for the normal $\mathrm{ACD}=0.05 \mathrm{~m}$ and the reduced $\mathrm{ACD}=0.037 \mathrm{~m}$ (lowered anodes) obtained when using the empirical bottom friction coefficient $\mathrm{Cf}=0.025$.

\section{Conclusions}

The specialised MHD modelling software MHD-VALDIS is relatively easy to set up for a variety of the commercial cells accounting for the industry specific requirements needed to gain understanding of the existing cell performance and limitations. The measurements provided by the RUSAL team are replicated by the numerical modelling to a reasonable accuracy, predicting the velocity fields, the stationary liquid metal shape, wave development and the stability limits.

\section{References}

[1] V. Mann, V. Buzunov, N. Pitertsev, V. Chesnyak and P. Polyakov, Reduction in Power Consumption at UC Rusal's Smelters 2012-2014, Light Metals (2015), 757-762. 
[2] M. Dupuis, ANSYS-Based 3-D Thermo-Electric Heat Balance Models, Genisim Brochure, http://www.genisim.qc.ca/download/3dmodelsadd.pdf

[3] A. Alzarooni, L. Mishra, M. Bastaki, A. Al Jasmi, A. Arkhipov and V. Potocnik, Mathematical model validation of aluminium electrolysis cells at DUBAL, Light Metals (2013), 597-602.

[4] Liu Wei, Zhou Dongfang, Liu Yafeng, Liu Ming, Yang Xiaodong, Simulation and Measurements on the Flow Field of 600ka Aluminum Reduction Pot, Light Metals (2015), 479-482.

[5] M. Baitece, S.M. Taghavi, D. Ziegler, M. Fafard, LES Turbulence Modeling Approach for Molten Aluminum and Electrolyte Flow in Aluminium Electrolysis Cell, Light Metals (2017), 679-686.

[6] H. Zhang, J. Liang, J. Li, K. Sun, J Xiao, Evolution of the Busbar Structure in Large-Scale Aluminum Reduction Cells, JOM (2017), 69 (2).

[7] V. Potocnik and F. Laroche, Comparison of Measured and Calculated Metal Pad Velocities for Different Prebake Cell Designs, Light Metals (2001), 419-425.

[8] A. Arkhipov, A. Alzarooni, A. Al Jasmi and V. Potocnik, Improving the Understanding of Busbar Design and Cell MHD Performance, Light Metals (2017), 671-677.

[9] V. Bojarevics and J.W. Evans, Mathematical Modelling of Hall-Herault Pot Instability and Verification of Measurements of Anode Current Distribution, Light Metals (2015), 783-788.

[10] V. Bojarevics, Time Dependent MHD Models for Aluminium Reduction Cells, Light Metals (2010), 199-206.

[11] V. Bojarevics and K. Pericleous, Solution of the Metal-Bath Interface for Aluminium Electrolysis Cells, Light Metals (2009), 569-574.

[12] J. Zoric, J. Thonstad and T. Haarberg, The Influence of the Initial Shape and Position of an Anode and the Curvature of the Aluminium on the Current Distribution in Prebaked Aluminium Cells, Met. Trans. (1999), 30B, 341-348.

[13] M.A. Cooksey, M.P. Taylor and J.J.J. Chen, Resistance Due to Gas Bubbles in Aluminium Reduction Cells, JOM (2008), February, 51-57.

[14] R. Beeler, Bar to Block Contact Resistance in Aluminum Reduction Cell Cathode Assemblies, Light Metals (2014), 507-510.

[15] J. Thonstad and H. Vogt, Terminating Anode Effects by Lowering and Rising the Anodes, Light Metals (2010), 461-466.

[16] B.F. Bradley, E.W. Dewing, J.N. Rogers, Metal Pad Velocity Measurements by the Iron Rod Method, Light Metals (1984), 541-552. 